\title{
LA SABIDURÍA COMO FUNDAMENTO DE UNA EXISTENCIA ÉTICA Y FRATERNA EN LA TEOLOGÍA PAULINA
}

\section{WISDOM AS FOUNDATION FOR A FRATERNAL AND ETHICAL EXISTENCE IN THE PAULINE THEOLOGY}

Emerson Rodríguez Delgado1

\begin{abstract}
RESUMEN
Pablo cambia su presentación de la revelación de la sabiduría divina desde un tono paradójico en 1Cor 1,17-2,5 hacia uno más confrontacional y revelatorio en 1Cor 2,6-16, a fin de dar los elementos necesarios a los creyentes para discernir la realidad dinámica de su relación con el "voṽ Xeıбoṽ" a través del Espíritu. Esta presencia pneumática desde su recepción en el Bautismo otorga al creyente una nueva identidad ontológica. De esta manera, los creyentes, "los que aman a Dios -los maduros," son quienes comunican la realidad salvífica de Dios en su manifestación cruciforme de la sabiduría divina en Cristo. Por tanto, un creyente "maduro" vive de acuerdo a su nueva identidad cuando acoge y acepta el "logos de la cruz" a través de una escucha atenta y lo traduce en una vida pneumática (según el Espíritu), que discierne y reconoce la realidad estaurológica y kenótica del Evento-Cristo en medio de la comunidad, la que se cristaliza en una existencia ética y fraternal con los demás.
\end{abstract}

\section{PALABRAS CLAVE:}

Sabiduría, Espíritu, revelación, maduros/perfectos, pneumáticos, psíquicos, discernir

\begin{abstract}
Paul shifts his presentation of the revelation of divine wisdom, from a paradoxical tone in 1Cor 1,616 to a more confrontational and revelatory tone in 1 Cor 2,6-16, in order to provide the necessary

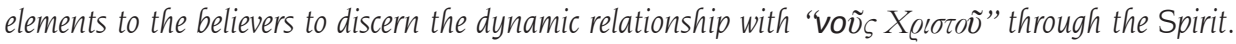
From his reception in the Baptism, the believer is granted a new ontological identity by this pneumatic presence. In this fashion, the believers, "who love God - the mature/perfect," communicate God's salvific reality in his cruciform manifestation of Divine wisdom in Christ. Therefore, a "mature/perfect" believer lives according to his new identity when takes in the "logos of the cross" by an attentive listening and rendering into a pneumatic life that discerns and acknowledges Christ-event' staurological and kenotic reality amidst the community, which is materialized in an ethical and fraternal existence with others.
\end{abstract}

\section{KEYWORDS:}

Wisdom, Spirit, revelation, mature/perfect, pneumatics, psychics, discern

* Docente de la Universidad Femenina del Sagrado Corazón: emersonrodriguez@me.com 


\section{INTRODUCCIÓN}

El presente estudio exegético girará en torno a la comprensión paulina de la "sabiduría" en 1 Cor 2,6-16. Donde se profundizará cómo Pablo despliega su discurso deliberativo a fin de definir, precisar y distinguir la naturaleza, la comunicación y la aplicación pragmática de la sabiduría entre los creyentes respecto a la sabiduría humana y mundana. Así, Pablo tratará de deslindar su enfoque cruciforme de la sabiduría divina respecto de la judío-helenista y de las religiones mistéricas. Para esto, comienza por su comprensión de la sabiduría divina por medio de una serie de contrastes "correctio" con la sabiduría mundana (vv. 6-9). Para luego, presentar el rol del $\pi v \varepsilon \tilde{\mu} \mu \alpha$ para obtener la sabiduría divina (vv. 10-12). 廿uxıxòs y $\pi v \varepsilon u \mu \alpha \tau \iota x o ̀ s ~(v v .13-16)$. En otras palabras, para identificar quiénes están viviendo de acuerdo a su nueva identidad como creyentes en este proceso dinámico

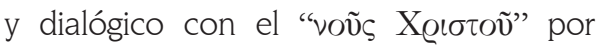
medio del $\pi v \varepsilon \tilde{v} \mu \alpha$.

El desarrollo de este análisis del texto constará, primero, de una justificación del texto como unidad literaria. Luego, un estudio de la estructura literaria, la que examinará su unidad y coherencia lingüística, sintáctica y temática. Asimismo, su progresión literaria en la presentación de la revelación de la sabiduría misteriosa. Finalmente, una parte exegética detallada de los elementos más significativos para la comprensión de la perícopa.

\section{JUSTIFICACIÓN DE LA PERÍCOPA (1 COR 2,6-16)}

- La presencia de nuevos sujetos en el desarrollo de la argumentación a partir del 2,6 "NOSOTROS" con relación al "YO - USTEDES" tanto del 2,1 5 como del 3,1 4.
- Cambio del tiempo verbal al "presente" a partir de 2,6 respecto a 2,1-5 en el "aoristo."

- En 1 Cor 2,6-16 encontramos un cambio en la línea temática respecto a la acción anterior de 1,18-25. Así, en 1 Cor 2,6-16, el eje temático gira alrededor de la revelación de la sabiduría divina por medio del Espíritu y sus consecuencias. Mientras que, en 1 Cor 1,18-25 los temas ejes son el anuncio del Cristo crucificado y la sabiduría del mundo.

- Cambio en la función que cumple la sabiduría a lo largo del discurso. En 1 Cor 2,6-16, la sabiduría se convierte en el objeto del discurso, es decir, puede ser conocida y comunicada mientras que, en su antecedente inmediato cumple una función meramente instrumental (discurso según criterios humanos de sabiduría)

- Con el enunciado en 1 Cor 2,6 "Entre maduros (o a los maduros) comunicamos una sabiduría" se abren las puertas hacia un nuevo movimiento argumentativo progresivo que culmina con un epifonema en 2,16b (MORTARA GARAVELLI, 2003: 250-51), como vértice y síntesis de toda la argumentación de la unidad.

- Cambio en la orientación paulina de la probatio a partir de 1 Cor 2,6 desde una paradoxal e hiperbólica hacia otra con matíz positivo y asertivo. Es decir, en esta unidad de 1 Cor 2,6-16, la sabiduría de Dios manifestada en la locura de la cruz, es posible reconocerla y comunicarla gracias a la revelación del Espíritu. Así pues, los creyentes adquieren la capacidad para poder discernir y reconocer el proyecto sapiente-misterioso de Dios en el mundo, gracias a 
la mediación del Espíritu y a su continua presencia relacional en medio de la comunidad.

- Asimismo, el v. 6 constituye una sub-propositio, desde una aproximación del discurso retórico argumentativo. Puesto que éste retoma el tema de la sabiduría de la propositio principal de la unidad, a fin de desarrollar y completar lo afirmado en el v. 1,17

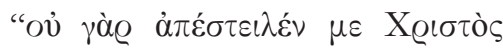

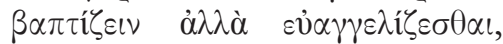

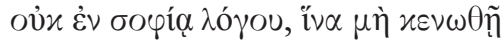

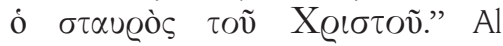
mismo tiempo, la presencia de una corrección sirve de conexión estilística para enlazar la propositio con la sub-propositio en 2,6. Además, esta figura cumple una función hermenéutica a fin de comprender, cómo el discurso procede por corrección, precisión y clarificación semántica sucesiva.

- Cambio del sujeto verbal a partir de 3,1. A saber, el paso del "NOSOTROS" de 2,6-16 al "YO-USTEDES" de 3,1-4. Por ende, esto significa que se produce un cambio en el plano comunicativo y argumentativo del discurso. Mientras que, en 2,616. Pablo afirma "entre maduros comunicamos..." en 3,1 "no he podido comunicarles porque no son maduros..." De modo que, Pablo salta del plano horizontal comunicativo del NOSOTROS a un plano de relación vertical "YO-USTEDES." De esta manera, Pablo toma distancia respecto a sus oyentes para confrontarlos en su situación real en la que viven. A saber, una situación en abierta disonancia con lo que significa ser maduros y espirituales. Y por consiguiente, Pablo los califica de carnales e infantes.

- Existencia de una composición literaria tripartita de tipo oral concéntrica en 1 Cor 2,6-16: A (69)-B (10-12)-A' (13-16).

- Por último, tanto en 1,26-2,5 como 3,1-4 cumplen una misma función de justificar las afirmaciones hechas en sus respectivas secciones antecedentes. A saber, la primera respecto a $1,18-25$ y la última, con 2,6-16. Con la salvedad que en 3,1-4, la situación de los Corintios está en una abierta disonancia con la de 2,6-16. 


\section{ESTUDIO DEL TEXTO}

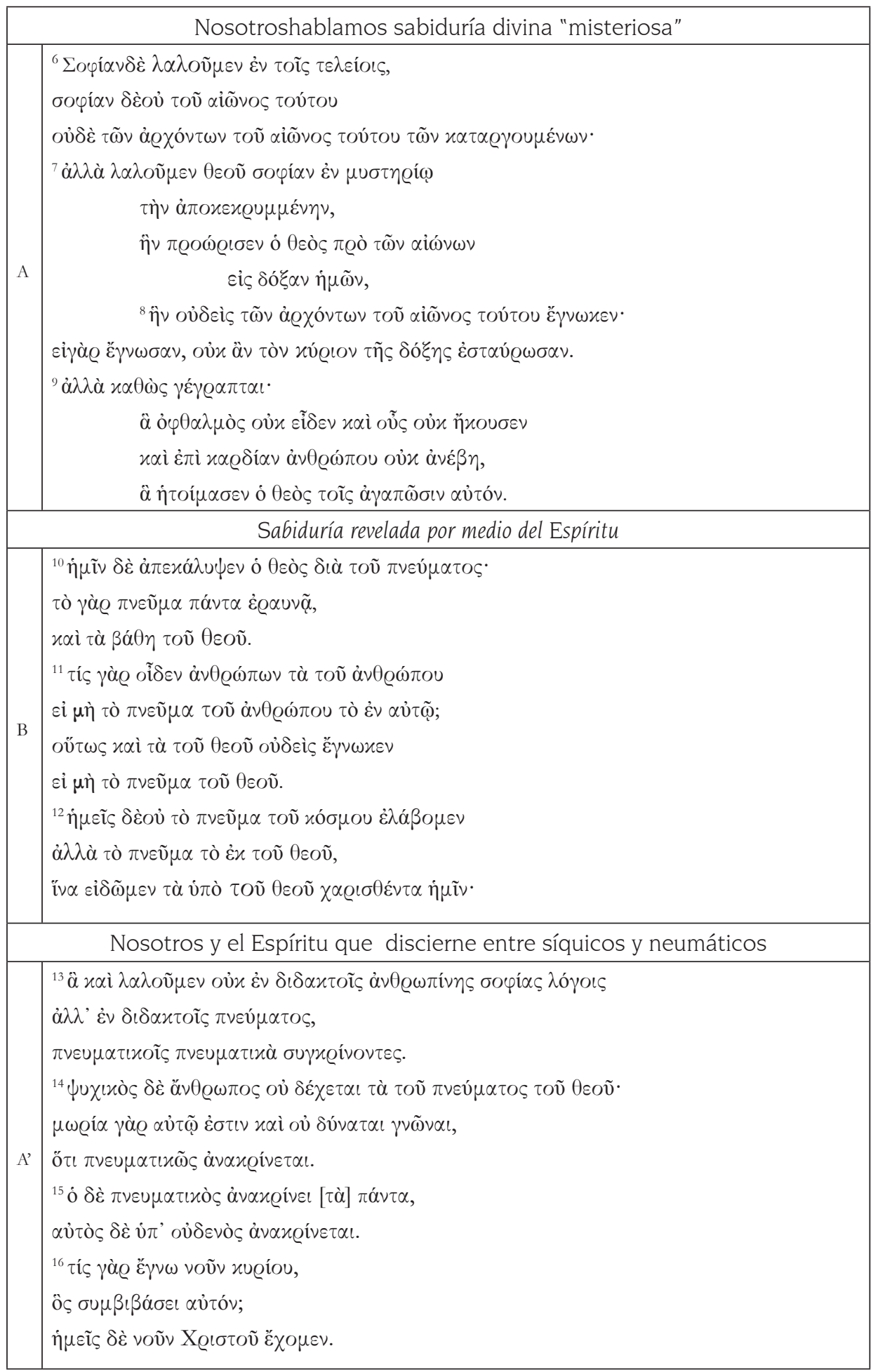




\subsection{Estructura Literaria}

En cuanto a 1 Cor 2,6-16, evidenciamos su coherencia literaria tanto en su estructura interna como en su relación con el conjunto de 1 Cor 1-4 a través del estudio de su disposición. Otros defienden un esquema conceptual binario de revelación en 2,6-16: "sabiduría escondida" - "sabiduría revelada."

1 Cor 2,6 constituye una sub-propositio (La función de la sub-propositio consiste en desarrollar progresivamente algunos elementos claves de la propositio principal, y a su vez, de introducir nuevas fases de argumentación (cf. ALETTI, 1992, NTS 38, 385-401) respecto a la propositio principal en 1,17 de toda la unidad de 1 Cor 1-4 cuyos temas centrales son: la cruz de Cristo, el anuncio del Evangelio, y la sabiduría. Tanto las sub-propositio de 1,18 como de 2,6 mantienen una relación de continuidad y novedad respecto a la propositio de la cual dependen. Por ejemplo, en el caso de 2,6 retoma el tema de la sabiduría para darle un desarrollo posterior a través de precisiones sucesivas (sabiduría de Dios-sabiduría en misterio, sabiduría escondida, etc.). Además, 2,6 se enlaza estilísticamente con 1,17 a través de una correctio + un paralelismo semántico. Al mismo tiempo, notamos un cambio en los protagonistas del discurso con respecto a 2,5. Y por último, a partir de 3,1 , evidenciamos una ruptura en el tono discursivo y en el plano comunicativo.

En adelante, comenzamos a deshilvanar el texto teniendo en cuenta primero su composición literaria, la cual se trata de una típica composición paulina que corresponde al modelo oral circular tripartito: A (6-9) - B (10-12) - A' (13-16).

La primera sub-unidad de los vv. 6-9 enuncia el tema central a través de una correctio en el v. 7, a saber, la sabiduría de Dios permanece escondida e incognoscible para el mundo. Es más, el v.9 confirma tal realidad recurriendo a un pasaje de la escritura.

A nivel lexical, el verbo " $\lambda \alpha \lambda$ oṽ $\mu \varepsilon \nu$ "es usado dos veces en los vv. 6.7. De esta manera, el sujeto verbal principal es NOSOTROS (Pablo y sus oyentes). Del mismo modo, dilucidamos varios puntos de contacto entre 2,6 con el posterior desarrollo de la unidad. Por ejemplo, la primera persona plural, "sabiduría", "eón", "jefes, líderes." Por último, entran en escena los tres actores principales: la sabiduría (6.7.8), Dios (7.9) que es el actor principal, y los dominadores de este mundo (7.8).

Por otro lado, los vv. 6-8 con el v.9 (la cita escriturística) se reclaman mutuamente porque ambos bloques expresan la misma realidad, a saber, la incognoscibilidad de la sabiduría de Dios para el mundo y sus dominantes, empero ésta ha sido pre-ordenada y revelada por Dios a los creyentes (maduros, que aman a Dios).

Asimismo, los vv. 6-9 se enlazan con la sub-unidad de los vv. 13-16, en cuanto que esta última desarrolla algunos de los elementos introducidos en los vv. 6-9 ( $\sigma \circ \varphi i \alpha, \tau \dot{\varepsilon} \lambda \varepsilon \iota \circ, \lambda \alpha \lambda \dot{\varepsilon} \omega$, etc.).

Ahora bien, en la segunda subunidad (10 12), el v. 10 enuncia el tema: la sabiduría revelada. La que comienza el desarrollo de una nueva unidad, cuyo tema dominante será el rol del Espíritu hasta el v. 16. De esta manera, inicia una nueva fase argumentativa con la entrada en escena del Espíritu. De modo que, en esta sección se presenta la revelación de Dios a través del Espíritu y la modalidad de éste. En adelante, encontramos a los dos actores principales: Dios, 
quien tiene presencia relevante en esta sub-unidad, y el Espíritu. Además, el uso de dos preguntas retóricas, en las dos sub-unidades en los vv. 11 y 16 respectivamente. En la primera, que da lugar a un razonamiento generalizado, de principios y de ejemplaridad. Y en la última en el v. 16 genera la síntesis de toda la argumentación de la unidad a través de un epifonema.

Por último, en los vv. 13-16, los tiempos verbales se encuentran al presente. Asimismo, retoman

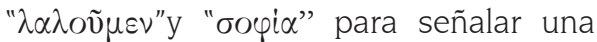
estructura composicional. Es por esto que, Pablo pone en relación la primera con la última sub-unidad. Al mismo tiempo, se encuentra la presencia de dos nuevos sujetos en una abierta relación antitética entre el hombre psíquico con el hombre espiritual. Y éstos, se definen como tal en cuanto a su capacidad de acoger la realidad del Espíritu. Igualmente, otra novedad, la constituye la presencia del campo semántico de los compuestos

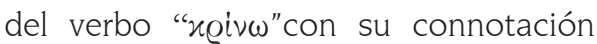
semántica de juicio, discernimiento, etc.

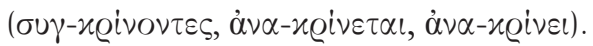

En cuanto a un análisis sintáctico del texto, encontramos en la primera subunidad de los vv. 6-9, que los vv. 6-7 se concatenan con la conjunción adversativa

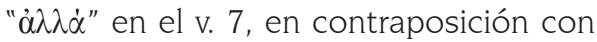
las partículas negativas "oủ" y "oủ $\delta \dot{\varepsilon}$ " del v. 6. Es decir, con la figura de pensamiento de la correctio. Esta sirve como medio para una clarificación semántica(cf. MORTARA GARAVELLI, 240). Mientras que los vv. 7-8 se enlazan a través del pronombre relativo femenino "ท๊v."

El segundo pasaje de los vv. 1012 se entretejen con una cadena de conjunciones que definen el cómo de la revelación $(\gamma \dot{\alpha} \varrho, \gamma \dot{\alpha} \varrho \ldots \varepsilon \dot{\imath} \mu \dot{\eta}$, ov̉... $\dot{\alpha} \lambda \lambda \dot{\alpha}$, íva). Así mismo, el v. 12 explicita el v.
10 en cuanto que el Espíritu revela la sabiduría divina a fin de que podemos

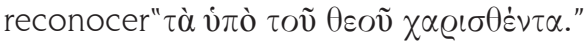

Así mismo, la concatenación entre la primera y la última sub-unidad se da en razón no sólo de las referencias lexicales del verbo" $\lambda \alpha \lambda \dot{\varepsilon} \omega$ " y de los sustantivos

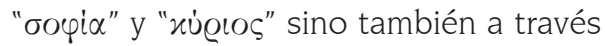
del uso de líneas sintácticas comunes a ambas como el uso del acusativo, que especifica el objeto del que se habla, más el verbo " $\lambda \alpha \lambda o v \tilde{\mu \varepsilon v " ~ m a ́ s ~ u n a ~ c o r r e c t i o ~}$ que desarrolla el discurso.

Por otro lado, el núcleo del texto lo encontramos en los vv. 10-12, que constituye al mismo tiempo el centro espacial de la unidad y el centro semántico de todo el texto (aunque este criterio no siempre es coherente). Además, por los reclamos lexicales entre los vv. 6-9 y vv. 13-16. Igualmente, en su línea sintáctica común: el verbo

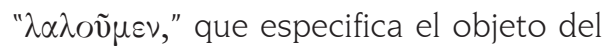
que se habla. Así, con el uso del verbo y una correctio, Pablo va desarrollando su discurso por medio de clarificación semántica progresiva.

Es más este núcleo sirve de clave de lectura para el paso de una sabiduría de Dios misteriosa y escondida al mundo (vv. 6-9) a la capacidad de los espirituales de poder discernir, reconocer y comunicar el proyecto salvífico de Dios en Cristo crucificado, quien es al mismo tiempo, la sabiduría de Dios (vv. 13-16). Es así que, este paso no sería posible sin la presencia de la sub-unidad central, que nos da la clave hermenéutica de todo el texto, a saber, la revelación gratuita de Dios y la acción mediadora del Espíritu entre Dios y los creyentes.

A modo de conclusión, podemos inferir que en los discursos Paulinos encontramos normalmente un esquema 
literario tripartito en el que la parte central provee los elementos claves y necesarios que permiten pasar de presupuestos indicados en la parte inicial a la conclusión de la parte final. Igualmente, hemos comprobado la existencia de una coherencia literaria en el texto tanto a nivel estilístico, terminológico y conceptual. Estilísticamente, con el uso de una cadena de correctio, y léxicamente, con varios términos que atraviesan todo el desarrollo del texto:

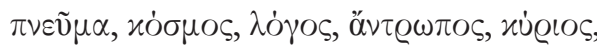
$\lambda \alpha \lambda \dot{\varepsilon} \omega$, etc. Por último, hay que precisar que el lenguaje que usa Pablo en este texto no pertenece al mistérico sino al apocalíptico, evidenciando por el uso de

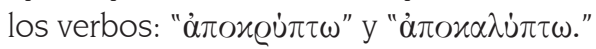

\subsection{Examen de las diversas progresiones retóricas del pasaje}

Examinar el modelo argumentativo subyacente al texto nos ayudará a comprender el modo a través del cual Pablo demuestra la tesis. Para esto, en primer lugar, es necesario localizar la propositio (un enunciado breve o a veces una expresión estilística no siempre uniforme como una tesis o pregunta, seguida de un desarrollo que la explica o justifica).

En nuestro caso será encontrar la sub-propositio. En el v. 6 evidenciamos su nexo sintáctico con la propositio principal en 1,17 con una correctio. Al mismo tiempo, ésta concatena con el v. 7. Además, a nivel literario existen varias conexiones con los versículos siguientes, por ejemplo, la primera persona plural, sabiduría, eón, jefes, líderes. Por otro lado, expone dos núcleos temáticos: a) maduros, quienes comunican una sabiduría (divina), y b) Lenguaje apocalíptico, que no pertenece al mundo presente ni a aquellos que lo dominan o guían. Además, con la presencia de la partícula adversativa " $\delta \varepsilon ́$ " con una carga enfática contrapone el discurso sobre la sabiduría de 2,6 16 (discurso revelatorio) con la sección precedente (discurso paradójico). Así pues, el apóstol señala un giro decisivo en el progreso de la argumentación respecto a lo afirmado anteriormente sobre la sabiduría de Dios "misteriosa" en el v. 7.

Sobretodo, la disposición literaria muestra los dos ámbitos en los que se moverá el desarrollo temático del discurso. Esto es, en el marco de una relación positiva entre sabiduría y maduros en el v. 6a, y otra negativa, entre la sabiduría y el eón presente con sus dominadores en el v. 6b.

Ahora analizaremos cómo se dan las progresiones en estos tres bloques que conforman el texto de 1Cor 2,6-16:

\subsubsection{Primer movimiento: Dimensión} de incognoscibilidad de la sabiduría divina en los vv. 6-9.

- El v. 6, la sub-propositio enuncia la tesis a demostrar o desarrollar. Es decir, una sabiduría comunicada entre maduros que no pertenece a este mundo o eón.

- En el v. 7, sabiduría que proviene de Dios y pertenece a Dios.

- Sabiduría misteriosa

- Sabiduría que permanece escondida

- Sabiduría pre-ordenada antes de los siglos

$\checkmark$ Prueba 1 (v. 8): Argumento de la experiencia: crucifixión de la sabiduría divina (cf. 1,30). Así, Pablo con la conjunción " $\gamma \dot{\alpha} \varrho$ "con 
valor explicativo, elucida el motivo por el cual el mundo y sus líderes fracasaron en reconocerla. El uso del condicional irreal confirma

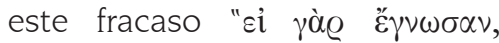

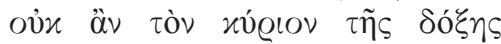

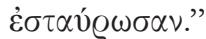

$\checkmark$ Prueba 2 (v. 9): Argumento de la autoridad de la Escritura: confirma esta realidad de incognoscibilidad de la sabiduría de Dios en misterio

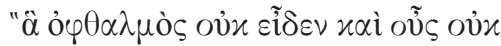

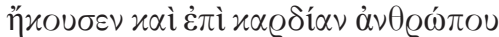

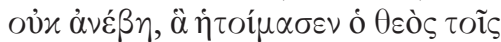
$\dot{\alpha} \gamma \alpha \pi \tilde{\omega} \sigma \iota \nu \alpha \hat{\tau} \tau$ ó."

\subsubsection{Segundo movimiento: El rol del} Espíritu en la función cognoscitiva en los vv. 10-12.

- El v. 10 es otra sub-propositio que explica la propositio del v. 6 e introduce, además, un nuevo actor en escena: el Espíritu.

- Prueba 1 (v. 10b): Argumento del Principio: que el espíritu es el único capaz de escrutar la profundidad de Dios y al mismo tiempo, la revelación sólo puede darse a través de un mediador. Y con la partícula adversativa $\delta \dot{\varepsilon}$ seguida de una conjunción explicativa " $\gamma \dot{\alpha} \varrho$,"testimonia el énfasis en esta transición substancial, progresiva y novedosa en el discurso. De esta manera, el v. 10 explicita cómo esa sabiduría divina misteriosa y escondida al mundo viene manifestada en favor de los creyentes por el Espíritu. De modo que, se introduce así el tema de la revelación y de la función del Espíritu en la vida del creyente. Igualmente, evidencia que el v. 10 no explicita aquello de los vv. 7-9 sino que señala un paso decisivo a otra sección nueva.
- Prueba 2 (vv. 11-12):una ilustración, que sirve para hacer un parangón entre el espíritu del hombre, que conoce la realidad escondida del hombre, y el Espíritu de Dios, que escruta la profundidad misma de Dios. Además, se profundiza en las funciones del Espíritu y sus consecuencias para los creyentes.

1.2.3. Tercer movimiento: El rol del Espíritu en la revelación de Dios a favor de los creyentes en los vv. 13-16.

- El v. 13 retoma el verbo " $\lambda \alpha \lambda$ oṽ $\mu \varepsilon v . "$ Es decir, se vuelve al v. 6, al punto de partida, para arribar ahora a una conclusión que cierre el arco argumentativo empezado por la subpropositio en el v. 6. En otras palabras, se define el término maduros en el $\mathrm{v}$. 16. Además, el v. 13 tiene otro punto con el v. 6 a través de la sabiduría con la diferencia que en el v. 13, adquiere un valor instrumental por la preposición " $\dot{\varepsilon} \nu$ " que la precede. Por último, tanto en el v. 13 como el v. 6 presentan una misma estructura sintáctica a través del uso de la figura de la correctio: "oủx... $\dot{\alpha} \lambda \lambda \dot{\alpha}$." Con la observación que en el v. 6 presenta la primera parte de la negación "oủ" completada en el v. 7 con " $\alpha \lambda \lambda \dot{\alpha}$." Por

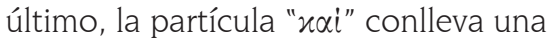
carga adversativa en consonancia con la progresión del pensamiento paulino del v. 12.

- En los vv. 14-15 a través de la contraposición entre hombre carnal y hombre espiritual hace referencia también al v. 6. Es decir, a los dominadores-líderes del mundo corresponde el sintagma nominal hombre psíquico y a maduros con hombres espirituales. Con respecto, a las razones sintácticas para leer v. 
13 con v. 14 la encontramos en el uso de contrastes. Así, en el v. 13 usa una correctio "ở $x$... $\dot{\alpha} \lambda \lambda \dot{\alpha}$." Con "oưx" empieza negando que la sabiduría entre los creyentes (madurosperfectos) se trasmita a través de las categorías Greco-romanas sino " $\alpha \lambda \lambda \dot{\alpha}$ " por medio de una experiencia carismática del Espíritu. Mientras que en el v. 14, Pablo usa una negación "oủ" seguida de " $\gamma \dot{\alpha} \varrho$ " para evidenciar la necedad de la sabiduría mundana al no aceptar la sabiduría divina cruciforme. Aquí " $\gamma \dot{\alpha} \varrho$ " es posible entenderlo como el equivalente a " $\delta \varepsilon \varepsilon^{\prime}$ Es decir, con el valor de una correctio. Además, de su valor causal y explicativo (cf. ZERWICK, 2005:159).

- En el v. 16, Pablo incrusta una cita bíblica como en la sección anterior de los vv. 6-9. Ésta cumple una función conclusiva del discurso, cargada de un tinte irónico, provocador y polémico con una pregunta retórica. La imagen a evocar podría ser como la del cierre intempestivo del telón en medio de una función teatral, a fin de generar un clima de suspenso y de un silencio inquisitivo, y asimismo, por parte del espectador, de estar a la expectativa de lo que en cualquier momento pueda surgir al abrir el telón. Y finalmente, cuando Pablo lo corre, responderá inmediatamente a la interrogante abierta hecha por la cita del A.T. con una respuesta nueva, inaudita e inesperada. A saber, "ĩ $\mu \varepsilon \tilde{i} \varsigma$

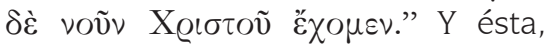
absolverá especialmente el suspenso

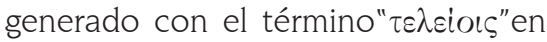
la propositio del v. 6.

En conclusión, el texto se desarrolla en tres momentos definidos por los criterios literarios y temáticos: vv. 6-9/10-12/13-16. Los que a su vez, constituyen también los tres desarrollos argumentativos de la propositio principal del v. 6 , la que es coordinada con la propositio secundaria en el v. 10. De modo que, pueden ser consideradas como tres momentos de la revelación del misterio (La presencia de un modelo teológico de revelación a lo largo de este texto. Donde el lenguaje apocalíptico cumple una función hermenéutica. Es decir, de discernir el proyecto sapiente de Dios realizado en Cristo. Acerca de este tema nos dará luz analizar Dan 2,27.30).

\section{Parte exegética}

En esta sección abordaremos los puntos más destacados en la exégesis del texto.

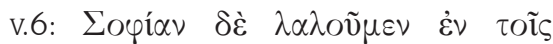

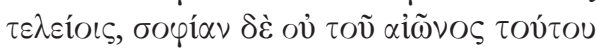

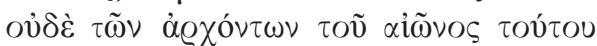
$\tau \tilde{\omega} \nu \varkappa \alpha \tau \alpha \varrho \gamma O u \mu \varepsilon \dot{\varepsilon} \nu \omega \nu$.

A partir de 1 Cor 2,6, Pablo abandona el tenor cristológico de 1,18-2,5 para concentrarse en el papel fundamental que asume el Espíritu en el proceso de la revelación divina.

Por otro lado, la sabiduría ya no es más asociada a la realidad estaurológica sino más bien que se pone en relación al plan de Dios en misterio y preordenado antes de la creación del tiempo (cf. 2,10.12). En adelante, Pablo evitará hablar de sabiduría debido a que ésta se presta a una ambigüedad y a una identificación con una sabiduría meramente humana o mistérica por el influjo de una cultura judío-helenística y de las religiones mistéricas (Por esa razón, Pablo es muy cuidadoso al usar el término de sabiduría en 2,7. Y lo usa como un sintagma preposicional para 
marcar las diferencias propias del caso). Por ende, Pablo preferirá de ahora en adelante el término de espirituales.

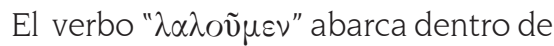
su rango semántico el de COMUNICAR (sabiduría). Además, para Pablo este verbo connota una dimensión de una relación dialógica con su comunidad. Es decir, la actitud de ponerse en el mismo plano que sus oyentes. Con esto Pablo, intenta generar una cercanía e intimidad con sus oyentes (una captatio benevolente). Por consiguiente, él procura que éstos también se sientan identificados con él. Para así, tener el campo más propicio y dar a conocer su mensaje. Esto lo podemos constatar en Rom 7,1; 15,18; 1 Cor 15,34, etc.

En resumen, Pablo usa la primera persona plural de este verbo para señalar el giro de su discurso hacia dentro de la comunidad, en el cual él se incluye a fin de interpelar a sus oyentes en una forma directa.

El sintagma preposicional «entre maduros» hace eco de un trasfondo cultural religioso tanto de un judaísmohelénico como de las religiones mistéricas. Así pues, maduros define a aquellos que junto con Pablo, disciernen, comprenden, y comunican la sabiduría divina. Al mismo tiempo, señala la identidad propia de cada cristiano. Por otro lado, Pablo deja aún la definición de maduros en suspenso hasta v. 16c. Pero, en el v. 9, Pablo nos da una característica de éstos, a saber, los

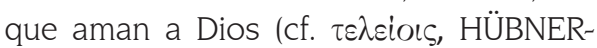
BALZ - SCHNEIDER, 1995, II: 1588-1592).

En el v. 7, el sintagma preposicional "en misterio" se refiere a la modalidad a través del cual la sabiduría se realiza y manifiesta (cf. Estilo elíptico de la construcción sabiduría divina que se realiza misteriosamente). Así, el sintagma preposicional califica la sabiduría divina de un modo decisivo (cf. ALETTI, 1995).

El lexema " $\delta$ ó $\alpha$, gloria" (cf. SPICQ, 1988, I: 418 436) ¿Cómo se interpreta este término? ¿Tiene un matiz escatológico? ¿Cuál es su relación de este término aplicado a "xủ@ıs"? ¿Cuál es la relación entre los vv. 9 y 10 ?

El campo semántico del término comprende: dignidad, gloria, esplendor, reputación y honor ( cf. 2Cor 3,9.10.11). Aquí, no está en juego el honor de la gloria futura. Es decir, una dimensión escatológica sino que por el contrario, mira a la situación presente de los cristianos, y a la honorabilidad del anuncio cristiano y la fidelidad a éste. Además, el término hebreo subyacente al griego " $\delta$ ó $\alpha^{\alpha}$ " es "ëáåã ( $\left.k a b o ̄ d\right)$, peso". De modo que, una persona "pesada" para la mentalidad semita supone que es una persona sabia, estable, confiable, noble, paciente, y con sano juicio (cf. BAILEY, 2011, p. 110).

En los vv. 10-12, se nota el uso de verbos que están dentro del rango semántico de conocer en sus diversas

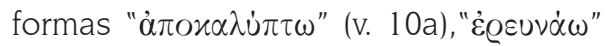

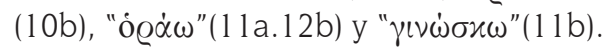

¿Cuál es la función del verbo

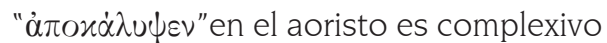
(cf. BLASS - DEBRUNNER - REHKOPF, $\oint$ 332; ZERWICK, §§253.254), continuativo (ZERWICK, $\oint \oint 242-249)$ o puntual?

¿Cuál es el alcance del término

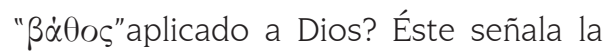
insondabilidad de Dios, ya sea de actuar, o de su plan de salvación por parte de toda sabiduría humana. Por consiguiente, el plan de Dios en misterio permanece inaccesible e inalcanzable a este mundo y a sus líderes por medio de la sabiduría humana (SCHRAGE, 257-258). 
¿Cuál es el campo semántico del

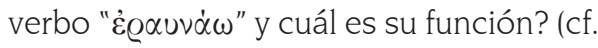
Sab $7,22 \sim 8,1)$.

¿Cuál es la función y el límite de la comparación en el v. 11 entre espíritu del hombre y Espíritu de Dios? (cf. GAFFIN, 1995: 103-124).

"Espíritu del hombre" es la autoconciencia que el hombre tiene de sí mismo (Cf. JEWETT, 1971: 167-175). Por ende, esto no se puede aplicar al Espíritu de Dios debido a que en el v. 12 viene descrito como realidad diversa a Dios, que escruta la profundidad de Dios, y a la vez, que se manifiesta fuera de él a los creyentes.

¿En qué sentido se puede comprender este uso bivalente de " $\pi \nu \varepsilon \tilde{v} \mu \alpha$ " tanto en espíritu del hombre como en Espíritu de Dios?

Pablo usa " $\pi v \varepsilon \tilde{v} \mu \alpha$ " tanto para Dios como para el hombre en virtud de la aplicación de una figura del lenguaje como la Antanáclasis. La que consiste en una repetición del término en sentido opuesto (cf. GARAVELLI, 214). De modo que, el mismo término puede ser aplicado tanto para el hombre como para Dios. $\mathrm{Y}$ esto por dos motivos, primero por analogía, para poner el espíritu como actor fundamental en relación con Dios, y por último, señalar su función mediadora entre Dios y los creyentes.

¿Cuál es la relación entre el "espíritu del mundo" y el Espíritu que procede de Dios? Este "espíritu del mundo" designa, más que un modo de pensar un estilo de vida arrogante y egocentrista propio de aquellas facciones en Corinto. Las que idolatraban la habilidad y astucia humana. A saber, la intelectual y competencia retórica por encima del estilo kenótico de Pablo en su anuncio del evangelio en clave estaurológica (1Cor 1,18-31). Así, los miembros de estas facciones confiaban en sus propias capacidades intelectuales para acceder a la sabiduría divina (cf. FRESTADIUS, 2011: 56)

¿A qué equivale el recibir el Espíritu (cf. Ga 3,2) para el creyente y qué matiz semántico encierra este verbo? Además, ¿Qué connotación semántica y teológica adquiere el verbo con el aoristo?

El verbo está en el aoristo pero no se refiere a un momento puntual del pasado sino que, por el contrario, a una situación tomada en su globalidad (cf. Rom 5,11). Es decir, es un aoristo complexivo. Por otro lado, recibir el Espíritu señala esencialmente una nueva identidad en los creyentes, que se genera a partir de la escucha atenta y a la acogida de la fe en el creyente. Además, se constituye, en el fundamento de una nueva relación con Dios a través del Espíritu. Igualmente este verbo resalta la dimensión de gratuidad de la iniciativa divina (cf. 1 Cor $4,7)$ de revelar su proyecto salvífico a los hombre maduros.

¿Cuál es el matiz y sentido de la preposición "દ̇x"? Esta preposición no indica sólo proveniencia sino también, un nivel de pertenencia. Es decir, el Espíritu pertenence al mundo de Dios, y de esta manera, se mueve en el mismo plano que Dios (THISELTON, 260-264).

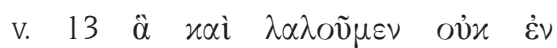

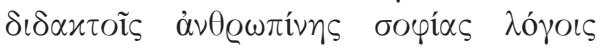

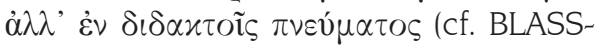

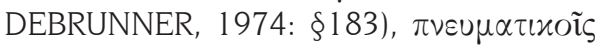
$\pi v \varepsilon v \mu \alpha \tau 1 \kappa \alpha ̀ ~ \sigma u \gamma \varkappa \varrho i ́, \nu O \nu \tau \varepsilon \varsigma$.

El v. 13 retoma el verbo " $\lambda \alpha \lambda$ oũ $\mu \varepsilon \nu "$ que presenta la modalidad neumática de comunicarse entre los creyentes a fin de remitirnos a la propositio en el v. 6. Al mismo tiempo, su sintáxis revela 
una conexión con el v. 12 a través de una correctio. Asimismo, se concatena a nivel de contenido con la presencia del pronombre relativo "ò,"el que hace

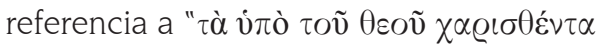
$\eta \dot{\eta} \tilde{\nu} . " ¿ A$ qué se refiere Pablo con

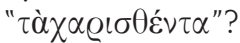

¿Cuál es el rango semántico del

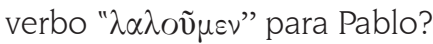

Primero que nada, Pablo usa este término no sólo para significar comunicación a un mero plano humano, sino también para referirse a esa expresión y comunicación del Espíritu, que da esa capacidad de reconocer y comprender el misterio de Dios a los maduros ó con los maduros (cf. DUPONT, 1949: 222226). Así pues, el apóstol nos presenta la modalidad neumática de comunicar a los creyentes, de la cual el mundo está excluído, con el uso de los dos sintagmas preposicionales "غ่v,"ambos con valor instrumental.

¿Cuál es la connotación semántica y

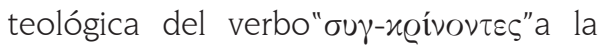
luz de los textos de Dan 5,12.14.16; Gen $41,12.13 .15$ y 2 Cor 10,12 ?

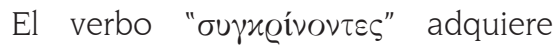
una connotación más precisa a la luz de los textos de Dan 5,12.14.16 y Gen 41,12.13.15. La connotación de este verbo es principalmente la capacidad de discernir, confrontar dos realidades, interpretar y explicar (cf. ELLIS, 1974: 128-144).

Así, se constata que la acción del Espíritu y presencia del Espíritu en los creyentes lejos de menoscabar las capacidades del hombre mas bien, las realza y potencia a su máxima capacidad humana para comprender y discernir el proyecto de salvación de Dios en la cruz (sabiduría divina misteriosa).
Cuál es el nexo entre " $\pi v \varepsilon u \mu \alpha \tau \varkappa$ ĩ

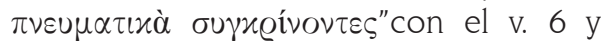
con 1 Cor 1,30? La figura etimológica consiste en la repetición de un vocablo en palabras contiguas o vecinas a fin de poner un énfasis semántico. Es decir, para reforzar y resaltar la importancia del término (cf. CARAVELLI, $210) . \dot{E} E s$ " $\pi \nu \varepsilon \cup \mu \alpha \tau \iota x o \tilde{\imath} \varsigma$ " masculino (personas espirituales) o neutro (cosas/ palabras espirituales), y cuál es la razón para esta elección?

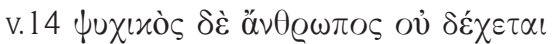

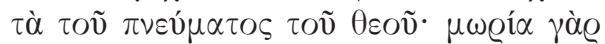

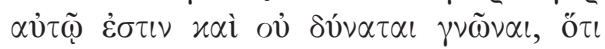

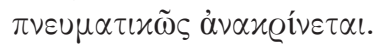

¿En función a qué criterios se define a los hombres como "భuxixòs, psíquicos" o " $\tau \nu \varepsilon u \mu \alpha \tau \iota x \tilde{\omega} \varsigma$, neumáticos"?

Los hombres se definen como "psíquicos" o "neumáticos" en relación a la acogida o rechazo del Espíritu (cf. Rom 6,6; Col 3,9-16; 2 Cor 4,16). Por consiguiente, el primero no acoge aquello que el Espíritu dona porque no lo puede reconocer. Es decir, esta no acogida o rechazo por parte de los primeros, conlleva a que no posean la capacidad para reconocer la cruz como evento salvífico, y la de una sabiduría de Dios que va más allá de los criterios sapienciales humanos.

Con respecto a la interpretación

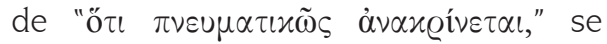
presta a varias posibles lecturas debido a la flexibilidad gramatical de la conjunción

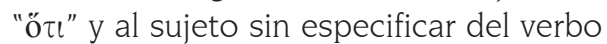

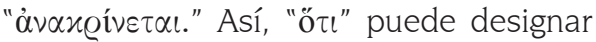
causalidad, pronombre relativo indefinido y la introducción de una proposición dependiente, la que a su vez, da pie a

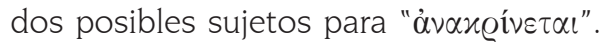

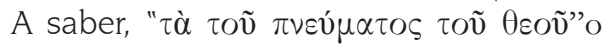

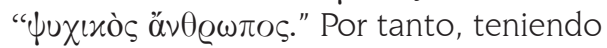


en cuenta el uso paulino de "ö $\iota "$ en sus cartas (cf. Rom 6,6; 2 Cor 8,9; 13,6; Flp 1,$12 ; 2,22 ;$ Ga 3,7), podemos optar por la última lectura cuyo sujeto sería "భuxıxò

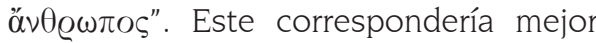
al paralelismo que se establece entre v. 14 "el hombre psíquico es examinado espiritualmente"con el v. 15 "el hombre espiritual no es examinado por nadie."

Ahora ¿cómo entendemos el rol - la relación del "hombre neumático" con el psíquico? ¿Qué tipo de categoría

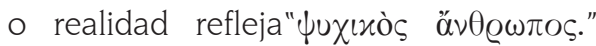
Es decir, ¿es una categoría estática o dinámica? En el v. 16 presenta al "neumático" como el creyente que

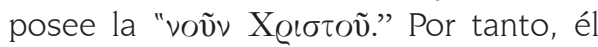
está equipado para examinar y discernir la situación de los psíquicos a fin de que ellos reconozcan su necedad, acepten la sabiduría divina misteriosa y vivan de acuerdo a su nueva identidad estaurológica en Cristo. Por consiguiente, el término psíquicos designa a aquellos que no están aun plenamente en Cristo (cf. DINGELDEIN, 2013: 31-44).

¿Cuál es el trasfondo teológico del

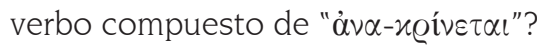

El verbo compuesto "ảv $\alpha-\varkappa \varrho i ́ v \varepsilon \tau \alpha \iota "$ (cf. 1Cor 14,24) comprende el rango semántico de investigar atentamente, escrutar y examinar la realidad a través del prisma de las categorías del Espíritu (La preposición "àv่' le da un plus semántico al verbo " $\varkappa i v \omega "$ en la medida que le añade la connotación de incrementar su capacidad de juicio y de juzgar la realidad en su totalidad (cf. Lidell \& Scott, 51-52). Y esto es posible, gracias a la presencia mediadora del Espíritu siempre presente en el creyente. Por ende, el Espíritu proporciona los criterios de la lectura de la cruz, los cuales nos llevan a reconocer el plan de salvación de Dios en el mundo. Por otro lado, aquellos que no tienen el
Espíritu no pueden reconocer ni acoger aquello que proviene del Espíritu (v. 14a).

¿Qué tipo de genitivo tenemos en la

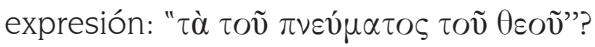

Identificamos un genitivo subjetivo, ya que, no es el Espíritu el que se da a conocer a sí mismo, sino aquello que el Espíritu revela al hombre, es decir, la salvación ofrecida por Dios al hombre a través de la cruz de Cristo.

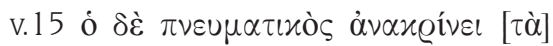

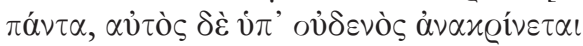

La partícula " $\delta \varepsilon \dot{~}$ cumple una función de poner énfasis en el contraste entre el hombre que piensa y actúa en un plano sólo humano con aquél que es espiritual.

El adjetivo " $\pi v \varepsilon u \mu \alpha \tau \iota x$ òs" clarifica que "maduros" son tales en cuanto a través de la obra y mediación del Espíritu tienen la capacidad de discernir y reconocer la sabiduría de Dios en el misterio de la cruz de Cristo.

El adjetivo " $\pi \alpha ́ v \tau \alpha$ " es un neutro acusativo, que se refiere al juicio sobre la realidad misma de Dios (no de los hombres), de sus criterios y de la manifestación de su designio en Jesús crucificado. "Juzgar toda cosa, toda realidad" ( $\pi \alpha ́ v \tau \alpha)$ como se afirma en el v. 10 «el Espíritu lo escruta todo» (cf. SCHANBEL, 2006).

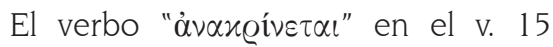
está en el presente, lo que significa que la capacidad de juzgar, discernir, etc. no es limitada a un tiempo puntual o a un acto concreto sino que instituye una nueva condición del creyente, un cambio de mentalidad y de juzgar las cosas que lo separa de la lógica del mundo.

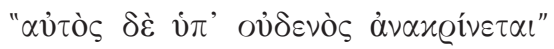
¿Cuál es la interpretación de la 
preposición de esta proposición?

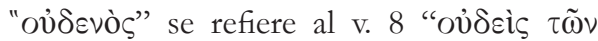

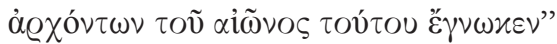

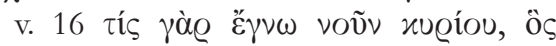

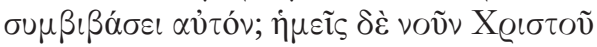

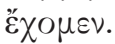

¿Cuál es la relación del v. 16a con las otras citaciones en las secciones precedentes? (cf. ELLIS, 1991: 22-25).

En esta oportunidad, Pablo sólo usa la partícula " $\gamma \alpha \grave{\varrho}$ "para indicar una transición, y al mismo tiempo, confirmar aquello que precede e introducir la cita bíblica. Así pues, Pablo deja de lado su estereotipada

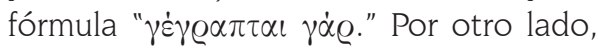
Pablo no concluye la argumentación con la cita bíblica, sino más bien que abre un nuevo desarrollo en vistas de una última precisión y actualización del sentido de esta cita bíblica tomada de Is 40,13. Por

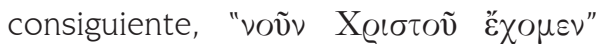
constituye el vértice de la argumentación y el cierre del arco argumentativo de los vv. 6-16.

¿A quién se refiere Pablo con "xú@ı๐s"a Dios o a Cristo?(cf. Is 40,13).

En esta unidad de 1Cor 2,6-16 se refiere a Dios en su misterio: "عै $\gamma \nu \omega$ voṽ xu@íou" = Dios.

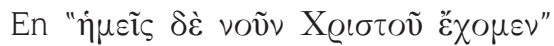

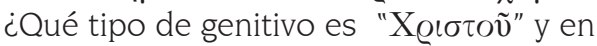
qué medida expresa la nueva identidad del creyente o es sólo un modelo a seguir?

Identificamos un genitivo epexegético. Es decir, "nosotros conocemos el "voũc" salvífico de Dios que es Cristo, el crucificado" (cf. 1 Cor 1,30). Por otro lado,

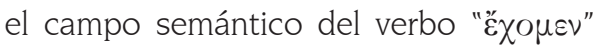
designa básicamente relación (cf. NIDA - LOUW, 1992). Por lo que, podemos concluir maduros (v. 6) - los que aman a
Dios (v. 9) - neumáticos (v. 15) reconocen y acogen el "voũs" salvífico de Dios por la revelación del Espíritu. De manera que, este reconocimiento y acogida indican una nueva identidad en el creyente que les inserta en una dimensión relacional con el "voũc" salvífico de Dios y la sabiduría de Dios misteriosa.

\section{CONCLUSIÓN:}

Pablo despliega su discurso argumentativo por medio de la presencia de varias correctio a fin de desacreditar el talante predominante de la sabiduría mundana en medio de la comunidad de Corinto. Quienes han adoptado los valores y categorías de la sabiduría helenista y de las religiones mistéricas, en vez de la sabiduría divina misteriosa manifestada paradójicamente en la realidad estaurológica y salvífica del evento-Cristo. De modo, que Pablo advierte que los corintios no están viviendo de acuerdo a su nueva identidad pneumática propia de creyentes como receptores del Espíritu en el Bautismo. Es así, que identifica como causa de todos los problemas y conflictos al interior de la comunidad como expresión de un rechazo de la realidad neumática. La cual se traduce en un riesgo inminente de perder la salvación y de mermar la capacidad de discernir y juzgar las cosas a la luz del Espíritu que capacita para entrar en una relación dialógica y amorosa con el "voṽ $\mathrm{X} \varrho \iota \tau$ เṽ."

\section{REFERENCIAS:}

ALETTI, J. N. (1992) La disposition rhetorique dans les epitres pauliniennes: propositions de method. En New Testament Studies, n. 38.

ALETTI, J. N. (1995) "Sagèsse et Mistère chez Paul". Association Catholique Française pour l'Étude 
de la Bible, La sagèsse biblique de l'Ancien aun Nouveau Testament. En lectio Divina, n. 160. Paris: Les Éditions du Cerf.

BLASS, F. - DEBRUNNER, A. REHKOPF, F. A. (1961) Greek Grammar of the New Testament and Other Early Christian Literature. Chicago: The University of Chicago Press.

BAILEY, Kenneth E. (2011) Paul Through Mediterranean Eyes. Cultural Studies in 1 Corinthians. Downers Grove, IL: InterVarsity Press.

BRANICK, Vincent P. (1974) Spirit and Knowledge: A Theological-literary Study of 1 Co 2, 6-12 and Related Pauline Texts. Roma: Pontificiolstituto Biblico.

DINGELDEIN, Laura B. (2013) "ö $\tau$

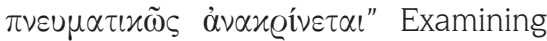
translations of 1 Corinthians. En Novum Testamentun, n. 55, pp. 31-44.

DUPONT, Jacques (1949) Gnosis: La Connaisance religieuse dans les Épitres de St. Paul. Louvain.

ELLIS, E. (1991) Paul's use of the O.T. London - Edinburgh: Baker.

ELLIS, E. (1974) Spiritual Gifts in the Pauline Community. En New Testament Studies, n. 20/2.

FESTADIUS, Simo (2011) The Spirit and Wisdom in 1Cor 2,1 13. En: JBPR, n. 3, pp. 52-70.
GAFFIN, R. B. (1995) Some epistemological reflections on 1 Cor 2,6-16. En Westminster Theological Journal, n. 57.

HÜBNER, H. - BALZ, H. - SCHNEIDER, G. (1995) Dizionario Esegetico del Nuovo Testamento. Brescia: Paideia.

JEWETT, R. (1971) Paul's Anthropological Terms. Leiden: Brill.

MORTARA GARAVELLI, B. (2003) Manuale de retorica. Milano: Bompiani.

NIDA, E. A. - LOUW,J. P. (1964) Lexical Semantics of the Greek of the New Testament. SBL; Atlanta, GA: Scholars Press.

SCHANBEL, E. J. (2006) Der Erste Brief des Paulus an die Korinther. HistorischTheologische Auslegung, Neues Testament. R. Brockhaus, Wuppertal.

SCHRAGE, Wolfgang (1999) Der Erste Briefan die Korinther.Patmos Verlag GmbH \& Co KG.

SPICQ, C. (1988) Note di lessicografia neotestamentaria. Paideia, Brescia.

THISELTON, A. C. (2000) The First Epistle to the Corinthians.New International Greek Testament Commentary. Eerdmans Publishing.

ZERWICK, M. (2005) Biblical Greek. Roma: Editrice Pontificio Istituto Biblico. 
